\title{
Parity, time since last live birth and long-term functional outcome: a study of women participating in the Norfolk Arthritis Register
}

\author{
E M Camacho, ${ }^{1}$ M Harrison, ${ }^{1}$ T M Farragher, ${ }^{1}$ M Lunt, ${ }^{1}$ D K Bunn, ${ }^{1,2}$ \\ S M M Verstappen, ${ }^{1}$ D P M Symmons ${ }^{1}$
}

- Additional data are published online only. To view these files please visit the journal online at (http://ard.bmj.com).

${ }^{1}$ Arthritis Research UK Epidemiology Unit, The University of Manchester, Manchester, UK

${ }^{2}$ Norfolk Arthritis Register, School of Medicine Health Policy and Practice, University of East Anglia, Norwich, UK

\section{Correspondence to} Deborah P M Symmons, Arthritis Research UK Epidemiology Unit, Manchester Academic Health Sciences Centre, University of Manchester, Stopford Building Oxford Road, Manchester M13 9PT, UK:

deborah.symmons@ manchester.ac.uk

Accepted 11 November 2010

\section{ABSTRACT}

Objective To investigate the relationship between pre-symptom onset live births and functional outcome in women with inflammatory polyarthritis (IP).

Methods 1872 women with no subsequent pregnancies were registered with the Norfolk Arthritis Register between 1990 and 2004 and followed-up for a median of 5 years. Functional disability over time was assessed by Health Assessment Questionnaire (HAO). The number and calendar year of past live births were recorded. Differences in $\mathrm{HAO}$ score over time by parity and time since last live birth (latency), adjusted for age and symptom duration, were examined using linear random effects models. The results were then adjusted for a number of potential confounders.

Results 1553 women (83\%) had $\geq 1$ live births before symptom onset. The median latency was 26 years (IOR 16-35). Parous women had significantly lower HAO scores over time than nulliparous women $(-0.19,95 \%$ $\mathrm{Cl}-0.32$ to -0.06$)$. Increasing latency was associated with increasing $\mathrm{HAO}$ score; the mean $\mathrm{HAO}$ score of women with a latency of approximately 32 years was the same as for nulliparous women. This was independent of autoantibody status, socioeconomic status, smoking history and comorbidity.

Conclusion Parous women who develop IP have better functional outcome over time than nulliparous women who develop IP. The beneficial effect of parity diminishes with time.

The incidence of rheumatoid arthritis (RA) among premenopausal women is approximately twice that in men. ${ }^{1}$ A number of studies have suggested that female reproductive events such as pregnancy or menopause may impact on a woman's likelihood of developing RA and subsequent disease severity. ${ }^{2-4}$ Some studies have identified nulliparity as being protective against developing RA, 56 some as a risk factor for RA, ${ }^{8}$ while other studies have failed to find a significant relationship. ${ }^{4} 9$ This discrepancy may be due to the difficulty in distinguishing between voluntary nulliparity and infertility. A cohort study of over 4 million people in Denmark reported no relationship between nulliparity or time since most recent birth and risk of RA. ${ }^{10}$

The relationship between disease outcome and pre-onset parity in RA has been less studied. A Dutch study of women with recent onset RA, aged 20-50 years at presentation, reported lower median 12-year Health Assessment Questionnaire (HAQ) scores for the 79 parous women compared with the
33 nulliparous women (1.0 vs 0.62 ), although this was not statistically significant and no adjustments were made for post-onset pregnancy. ${ }^{11}$

We have investigated whether pre-symptom onset parity (parous vs nulliparous) and latency (time between last live birth and rheumatoid symptom onset) are associated with functional outcome over time in a prospectively followed cohort of women with recent onset inflammatory polyarthritis (IP).

\section{METHODS}

Setting

The Norfolk Arthritis Register (NOAR) is a primary care-based inception cohort of patients with recent onset IP who presented to a physician with $\geq 2$ swollen joints lasting for $\geq 4$ weeks. Patients undergo a standardised assessment by a research nurse at baseline and years 1-5, 7, 10 and 15. NOAR has been described in detail previously. ${ }^{1}$

\section{Patients}

This investigation included all women who were registered with NOAR between January 1990 and December 2004 ( $n=1946$ ). Patients were followed to February 2009. Seventy-two women were excluded as they became pregnant during follow-up and two were excluded because the calendar years of their pre-onset live births were not recorded.

\section{Data collection}

At baseline, demographic, medical and reproductive history (before symptom onset) was recorded. Blood samples were taken and later tested for rheumatoid factor (RF) (latex method; positive titre $\geq 1: 40$ ) and anticitrullinated peptide antibodies (ACPA) (Axis-Shield DIASTAT kit; positive $>5$ units $/ \mathrm{ml}$ ). At each assessment patients completed the British HAQ. ${ }^{12}$ Blood samples were subsequently taken every 5 years, so the disease activity score (DAS) $28^{13}$ was calculated at these anniversaries only. The term 'parity' is used to describe whether patients ever had a live birth (ie, parous vs nulliparous). 'Latency' denotes the number of years between a patient's last live birth and symptom onset. Occupation was used to measure socioeconomic status (SES) using the UK Registrar General's system of occupation class; where not recorded, spouse's occupation was used. 'Number of comorbidities' was counted at the chapter level of the International Classification of Diseases Version 10 (ICD-10). 


\section{Statistical analysis}

Logistic and median regression adjusted for age at symptom onset was used to determine whether parity and latency were associated with the cohort characteristics at baseline. Linear random effects models were used to compare change in $\mathrm{HAO}$ score over time by parity and latency, adjusting for age and symptom duration (see online supplement for further details). Further adjustment was then made for SES, smoking history, autoantibody status, comorbidities and satisfaction of the 1987 American College of Rheumatology criteria for RA. ${ }^{14}$ All analyses were carried out using STATA Release 10 (StataCorp, College Station, Texas, USA).

\section{RESULTS}

Of the 1872 women in the cohort, 1553 (83\%) had at least one live birth before symptom onset. The median individual follow-up was 5.0 years (IOR 1.5-8.4) and total follow-up time was 11176 person years; 847 patients (45\%) met the 1987 ACR criteria for RA at baseline. Baseline characteristics are summarised in table 1. Parous women were less likely to be positive for ACPA or RF at baseline than nulliparous women, although this was not statistically significant. Baseline DAS28 and HAQ scores were comparable by parity. After adjustment for age at symptom onset, being parous was associated with a significantly lower baseline DAS28 score $(-0.27,95 \%$ CI -0.51 to -0.03$)$ but not $\mathrm{HAQ}$ score. The median time since last birth (latency) of the parous women was 26 years (IOR 16-35). Latency was not significantly associated with baseline DAS28 or HAQ score when adjusted for age at symptom onset (table 1 ).

Linear random effects analysis showed that, on average, parous women had lower HAQ scores at each follow-up anniversary than nulliparous women (mean difference $-0.19,95 \% \mathrm{CI}$ -0.32 to -0.06 ), adjusted for age, symptom duration and latency. As shown in figure 1 , the difference did not vary by anniversary. Baseline HAQ score increased by 0.06 ( $95 \%$ CI 0.02 to 0.10 ) per decade increase in latency. The relationship between parity and $\mathrm{HAQ}$ score diminished as the length of time since the last live birth increased. On average, only parous women who had a live birth within 32 years of symptom onset had lower HAQ scores than nulliparous women.
Additional adjustment for SES, smoking history, RF status, ACPA status, number of comorbidities and satisfaction of the 1987 ACR criteria for RA had little impact on these relationships (table 2).

Compared with nulliparous women, those with only one live birth before onset of symptoms had lower HAQ scores over time $(-0.14,95 \% \mathrm{CI}-0.30$ to 0.01$)$, as did women with $\geq 2$ live births before symptom onset $(-0.20,95 \%$ CI -0.33 to -0.06$)$, although the difference was only significant in women with $\geq 2$ live births.

\section{DISCUSSION}

In this cohort of women with recent onset IP, women who had one or more live births before symptom onset had less functional disability over time and milder disease activity shortly after symptom onset than nulliparous women. Among parous women, increasing interval between last live birth and symptom onset (latency) was associated with higher HAQ scores over time. Women with $\geq 2$ live births had the lowest HAQ scores.

Our findings are in keeping with those from the Dutch prospective study ${ }^{11}$ in reporting a better outcome for parous women. However, the Dutch study was smaller and their results were not statistically significant. It also included women with post-onset pregnancies and did not investigate the effect of latency. The relationship between latency and functional outcome found in our study, and the findings of a nationwide Danish study which reported that time since most recent birth was not associated with risk of RA development, ${ }^{10}$ suggest that the mechanism by which certain factors, such as this, determine the risk of disease development may differ from those which influence disease outcome.

Together with our previous report of a beneficial relationship between post-symptom onset pregnancies and functional outcome, ${ }^{15}$ these findings provide further evidence for a relationship between pregnancy and functional outcome in IP. By adjusting for a number of potential confounders, we have also demonstrated the robustness of this relationship.

Parity was associated with a significantly lower DAS28 score at baseline, but only significantly associated with HAO score

Table 1 Baseline characteristics by parity and association with parity (vs nulliparity) and latency (per decade increase in latency) adjusted for age at symptom onset

\begin{tabular}{|c|c|c|c|c|}
\hline Cohort characteristics at baseline & $\begin{array}{l}\text { Parous women } \\
(n=1553)\end{array}$ & $\begin{array}{l}\text { Nulliparous women } \\
(n=319)\end{array}$ & $\begin{array}{l}\text { Association with parity } \\
\text { (vs nulliparous) }\end{array}$ & $\begin{array}{l}\text { Association with latency } \\
\text { (per decade increase) }\end{array}$ \\
\hline \multirow[t]{2}{*}{ Median (IQR) age at IP onset (years) } & $54.3(44.6-65.2)$ & $54.1(36.4-70.0)$ & - & - \\
\hline & $n=1553$ & $\mathrm{n}=319$ & & \\
\hline \multirow[t]{2}{*}{ Median (IOR) symptom duration (months) } & $6.6(3.1-15.3)$ & $6.4(2.7-13.2)$ & - & - \\
\hline & $n=1553$ & $\mathrm{n}=319$ & & \\
\hline \multirow[t]{3}{*}{ Median (IOR) latency (years) } & $26(16-35)$ & - & - & - \\
\hline & $\mathrm{n}=1553$ & & & \\
\hline & & & $O R^{*}(95 \% \mathrm{Cl})$ & \\
\hline Met 1987 ACR criteria for RA, n/N (\%) & $704 / 1553(45.3)$ & $143 / 319(44.8)$ & $0.98(0.76$ to 1.26$)$ & $1.06(0.98$ to 1.14$)$ \\
\hline Positive for RF, n/N (\%) & $393 / 1370(28.7)$ & $90 / 262(34.4)$ & $0.76(0.57$ to 1.00$)$ & $0.93(0.86$ to 1.02$)$ \\
\hline \multirow[t]{2}{*}{ Positive for ACPA, n/N (\%) } & $388 / 1308(29.7)$ & $83 / 249(33.3)$ & $0.83(0.62$ to 1.11$)$ & $0.95(0.87$ to 1.04$)$ \\
\hline & & & Coefficient $†(95 \%$ CI) & \\
\hline \multirow[t]{2}{*}{ Median (IOR) DAS28 score } & $3.76(2.82-4.82)$ & $3.92(3.03-4.98)$ & $-0.27(-0.51$ to -0.03$) \ddagger$ & $-0.03(-0.10$ to 0.04$)$ \\
\hline & $\mathrm{n}=1240$ & $\mathrm{n}=230$ & & \\
\hline Median (IQR) HAO score & $\begin{array}{l}0.88(0.38-1.63) \\
n=1537\end{array}$ & $\begin{array}{l}0.94(0.38-1.63) \\
n=312\end{array}$ & $-0.08(-0.23$ to 0.08$)$ & $0.03(-0.02$ to 0.08$)$ \\
\hline
\end{tabular}

\footnotetext{
${ }^{*}$ Association of parity (vs nulliparity)/latency (per decade increase) with likelihood of meeting ACR criteria for RA or testing positive for RF or ACPA.

tChange in DAS28/HAQ score associated with parity (vs nulliparous)/latency (per decade increase).

$\ddagger$ Significant association.

ACPA, anticitrullinated peptide antibodies; ACR, American College of Rheumatology; DAS28, disease activity score; HAQ, Health Assessment Questionnaire; RA, rheumatoid arthritis; $\mathrm{RF}$, rheumatoid factor.
} 
Table 2 Results of linear random effects analysis: mean difference in HAO score over time by parity and association between $\mathrm{HAO}$ score and latency

\begin{tabular}{lll}
\hline Adjustment & Parous vs nulliparous (95\% CI) & Change in $\mathbf{H A O}$ score by latency (95\% Cl) \\
\hline Age at symptom onset, symptom duration & $-0.19(-0.32 \text { to }-0.06)^{*}$ & $0.06(0.02 \text { to } 0.10)^{*}$ \\
$\begin{array}{l}\text { As above plus SES, smoking history, RF status, ACPA status, comorbidity and } \\
\text { satisfaction of ACR criteria for RA }\end{array}$ & $-0.19(-0.34 \text { to }-0.05)^{*}$ & $0.05(0.002 \text { to } 0.09)^{*}$
\end{tabular}
satisfaction of ACR criteria for RA

Change in HAQ score by latency is per decade increase in latency.
Comorbidity is number of patient-reported comorbid conditions (by ICD-10 chapter).
Patients with RA met the 1987 ACR criteria for RA at least once during follow-up.
${ }^{*}$ Significant difference/change.
ACPA, anticitrullinated peptide antibodies; HAQ, Health Assessment Questionnaire; RF, rheumatoid factor; SES, socioeconomic status by occupation; smoking history at baseline (ever
smoked vs never smoked).

in our longitudinal analysis. Unfortunately, NOAR patients only donate a research blood sample every 5 years so we are unable to explore the association between parity and DAS28 score over time. Nevertheless, these results suggest that the lower HAO score over time in parous patients may be mediated by lower cumulative disease activity.

Fetal DNA circulating in the mother's bloodstream (microchimerism) may have a beneficial effect on rheumatoid symptoms, either by forcing the immune system to adopt a broader classification of 'self' - that is, which cells not to attack ${ }^{16}$ —or by contributing to tissue repair. ${ }^{17}$ Fetal cells may be detected in the maternal bloodstream for decades after birth, ${ }^{16}$ and the level of fetal DNA in the maternal bloodstream has been shown to correlate with disease activity. ${ }^{18}$ Decreasing microchimerism over time may explain the diminishing beneficial relationship between pregnancy and functional outcome with increasing latency. The level of genetic disparity between mother and baby may also be important; disparity in maternal-fetal HLA class II antigens has been shown to be associated with pregnancy-induced amelioration of symptoms in women with RA. ${ }^{19}$ However, a recent study reported that microchimerism of RA-associated HLA alleles is found more frequently in women with RA than in non-RA controls, ${ }^{20}$ and there is evidence to suggest that microchimerism is associated with the development of a number of autoimmune diseases. ${ }^{21}$

Another possibility is that pregnancy is associated with a delay in symptom onset resulting in milder long-term outcome. However, the age at symptom onset was very similar for the parous and nulliparous women in our cohort. Alternatively, nulliparity may be a risk factor for a poorer outcome in IP. Nulliparity may be a manifestation of infertility or subfertility in some women and this may be the consequence of adverse hormonal factors which, in turn, may influence a woman's risk of developing IP and its subsequent course. ${ }^{7}$ This is reflected in our finding that women with $\geq 2$ live births, potentially the most fertile, had lower HAQ scores than either nulliparous or monoparous women. In the context of our study, it is not possible to tell whether prior parity is protective against-or nulliparity is a risk factor for-more severe IP, or both.

This is the largest prospective study to investigate the relationship between pre-onset parity and functional outcome in a cohort of women with recent-onset IP or RA, and the first to investigate the role of latency. Our patients were recruited from primary care so there is a range of baseline disease severity represented in our cohort. The strengths and weaknesses of recruiting from primary care in NOAR have been discussed elsewhere. ${ }^{1}$ A potential weakness of this study is that we did not validate the patients' self-reported reproductive history. However, the event and calendar year of having a live birth are highly salient and likely to be remembered accurately. Another limitation of this study is that we were unable to investigate the impact of breast feeding, marital status or level of support received from offspring in our analyses. Further research is needed into pre-onset pregnancies with 'adverse' outcomes in women with IP, as it has been found that women with a history of adverse pregnancy outcomes are at greater risk of developing RA. ${ }^{22}$

In summary, we have shown that being parous is associated with better functional outcome in IP, the impact of which decreases as the length of time between a woman's most recent live birth and onset of symptoms increases.

Acknowledgements The authors acknowledge the support of clinical staff at the Norfolk and Norwich University Hospital and the local primary care physicians, as well as the data management team in Manchester.

Funding NOAR is funded by the Arthritis Research UK (grant reference 17552).

Competing interests None.

Ethics approval This study was conducted with the approval of the Norfolk and Norwich University Hospital research ethics committee.

Provenance and peer review Not commissioned; externally peer reviewed.

\section{REFERENCES}

1. Symmons DP, Barrett EM, Bankhead CR, et al. The incidence of rheumatoid arthritis in the United Kingdom: results from the Norfolk Arthritis Register. Br J Rheumatol 1994;33:735-9.

2. Da Silva JA, Spector TD. The role of pregnancy in the course and aetiology of rheumatoid arthritis. Clin Rheumatol 1992;11:189-94.

3. Jorgensen C, Picot MC, Bologna C, et al. Oral contraception, parity, breast feeding, and severity of rheumatoid arthritis. Ann Rheum Dis 1996;55:94-8.

4. Merlino LA, Cerhan JR, Criswell LA, et al. Estrogen and other female reproductive risk factors are not strongly associated with the development of rheumatoid arthritis in elderly women. Semin Arthritis Rheum 2003;33:72-82.

5. Silman AJ. Is pregnancy a risk factor in the causation of rheumatoid arthritis? Ann Rheum Dis 1986;45:1031-4.

6. Heliövaara $\mathbf{M}$, Aho K, Reunanen A, et al. Parity and risk of rheumatoid arthritis in Finnish women. Br J Rheumatol 1995;34:625-8.

7. Kay A, Bach F. Subfertility before and after the development of rheumatoid arthritis in women. Ann Rheum Dis 1965:24:169-73.

8. Spector TD, Roman E, Silman AJ. The pill, parity, and rheumatoid arthritis. Arthritis Rheum 1990;33:782-9.

9. Pikwer M, Bergström U, Nilsson JA, et al. Breast feeding, but not use of oral contraceptives, is associated with a reduced risk of rheumatoid arthritis. Ann Rheum Dis 2009;68:526-30.

10. Jørgensen KT, Pedersen BV, Jacobsen S, et al. National cohort study of reproductive risk factors for rheumatoid arthritis in Denmark: a role for hyperemesis, gestational hypertension and pre-eclampsia? Ann Rheum Dis 2010;69:358-63.

11. Drossaers-Bakker KW, Zwinderman AH, van Zeben D, et al. Pregnancy and oral contraceptive use do not significantly influence outcome in long term rheumatoid arthritis. Ann Rheum Dis 2002;61:405-8.

12. Kirwan JR, Reeback JS. Stanford Health Assessment Questionnaire modified to assess disability in British patients with rheumatoid arthritis. Br J Rheumatol 1986;25:206-9.

13. Prevoo ML, van 't Hof MA, Kuper HH, et al. Modified disease activity scores that include twenty-eight-joint counts. Development and validation in a prospective longitudinal study of patients with rheumatoid arthritis. Arthritis Rheum 1995;38:44-8.

14. Arnett FC, Edworthy SM, Bloch DA, et al. The American Rheumatism Association 1987 revised criteria for the classification of rheumatoid arthritis. Arthritis Rheum 1988;31:315-24.

15. Camacho EM, Farragher TM, Lunt M, et al. The relationship between post-onset pregnancy and functional outcome in women with recent onset inflammatory polyarthritis: results from the Norfolk Arthritis Register. Ann Rheum Dis 2010;69:1834-7 
16. Adams Waldorf KM, Nelson JL. Autoimmune disease during pregnancy and the microchimerism legacy of pregnancy. Immunol Invest 2008;37:631-44.

17. Nelson JL. Naturally acquired microchimerism: for better or for worse. Arthritis Rheum 2009;60:5-7.

18. Yan Z, Lambert NC, Ostensen M, et al. Prospective study of fetal DNA in serum and disease activity during pregnancy in women with inflammatory arthritis. Arthritis Rheum 2006;54:2069-73.

19. Nelson JL, Hughes KA, Smith AG, et al. Maternal-fetal disparity in HLA class II alloantigens and the pregnancy-induced amelioration of rheumatoid arthritis. N Engl J Med 1993;329:466-71.
20. Rak JM, Maestroni L, Balandraud N, et al. Transfer of the shared epitope through microchimerism in women with rheumatoid arthritis. Arthritis Rheum 2009;60:73-80.

21. Adams KM, Nelson JL. Microchimerism: an investigative frontier in autoimmunity and transplantation. JAMA 2004;291:1127-31.

22. Kaplan D. Fetal wastage in patients with rheumatoid arthritis. J Rheumatol 1986;13:875-7. 